\title{
Reversible Super-hydrophobicity to Super-hydrophilicity Transition of Aligned ZnO Nanorod Films
}

\author{
Xinjian Feng, Lin Feng, Meihua Jin, Jin Zhai, Lei Jiang, ${ }^{*}$ and Daoben Zhu \\ Center for Molecular Sciences, Institute of Chemistry, Chinese Academy of Sciences, Beijing 100080, P. R. China
}

Received September 21, 2003; E-mail: jianglei@iccas.ac.cn

Wettability is a very important property governed by both the chemical composition and the geometrical structure of solid surfaces. ${ }^{1}$ Super-hydrophobic surface (with water contact angle (CA) larger than $150^{\circ}$ ) and super-hydrophilic surfaces (CA close to $0^{\circ}$ ) have been extensively investigated due to their importance for industrial applications. ${ }^{2-6}$ Recently, with the development of smart devices, such as intelligent microfluidic switch, reversibly controlling the surface wettability has aroused great interest and been realized by modifying the surface with stimuli-responsive organic compounds. ${ }^{7}$ However, reversible switching between super-hydrophobicity and super-hydrophilicity has never been reported. Longterm durability is also difficult to be expected because of poor chemical, thermal, and mechanical stabilities of the organic compounds.

Being a wide band-gap semiconductor, $\mathrm{ZnO}$ possesses diverse range of high technological applications. ${ }^{8,9}$ However, nearly all of the applications are based on its optical, electronic, and acoustic properties. In this paper, controllable wettability of aligned $\mathrm{ZnO}$ nanorod films is reported. This inorganic oxide films show superhydrophobicity and super-hydrophilicity at different conditions, and the wettability can be reversibly switched by alternation of ultraviolet (UV) irradiation and dark storage. This effect is believed to be due to the cooperation of the surface photosensitivity and the aligned nanostructure of the films. Such special wettability will greatly extend the applications of $\mathrm{ZnO}$ films to many other important fields.

The aligned $\mathrm{ZnO}$ nanorods were synthesized via a two-step solution approach. First, $\mathrm{ZnO}$ sol, prepared according to the method of Sakohara, ${ }^{10}$ was spin cast onto a glass wafer several times and annealed at $420{ }^{\circ} \mathrm{C}$ to prepare a $50-100 \mathrm{~nm}$ thick film of crystal seeds. The sol-gel method was adopted here because of its simple facility and low cost. In particular, it derives films with preferential $c$-axial orientation, ${ }^{11}$ which is favorable for the subsequent growth of $\mathrm{ZnO}$ nanorods. Then the as-prepared wafer was suspended in an aqueous solution of zinc nitrate hydrate $(0.025 \mathrm{M})$ and methenamine $(0.025 \mathrm{M})$ at $90{ }^{\circ} \mathrm{C}$ for $3 \mathrm{~h}$, and it was removed from the solution, rinsed with deionized water, dried, and stored in the dark under clean air for several days. Figures $1 \mathrm{a}$ and $1 \mathrm{~b}$ are typical field-emission scanning electron microscope (FE-SEM) top-images of the as prepared films at low and high magnifications, respectively, showing a highly uniform and densely packed array of nanorods. The nanorods have flat hexagonal crystallographic planes projecting out of the crystal seeds layer, and their diameters are of 50-150 $\mathrm{nm}$. Figure 1c is a cross-sectional view of the sample, showing that the nanorods grow almost perpendicularly onto the substrates, and their lengths are about $1.2 \mu \mathrm{m}$. As confirmed by X-ray diffraction (XRD) with a remarkably enhanced (002) peak (Figure $1 d)$, the surface of the films is the (001) plane of the nanorods.

The wettability was evaluated by the water contact angle measurement of the as-prepared films. Figure 2a (right) shows a spherical water droplet with a water CA of $161.2 \pm 1.3^{\circ}$. Upon UV (obtained from a $500 \mathrm{~W} \mathrm{Hg}$ lamp with a filter centered at 365
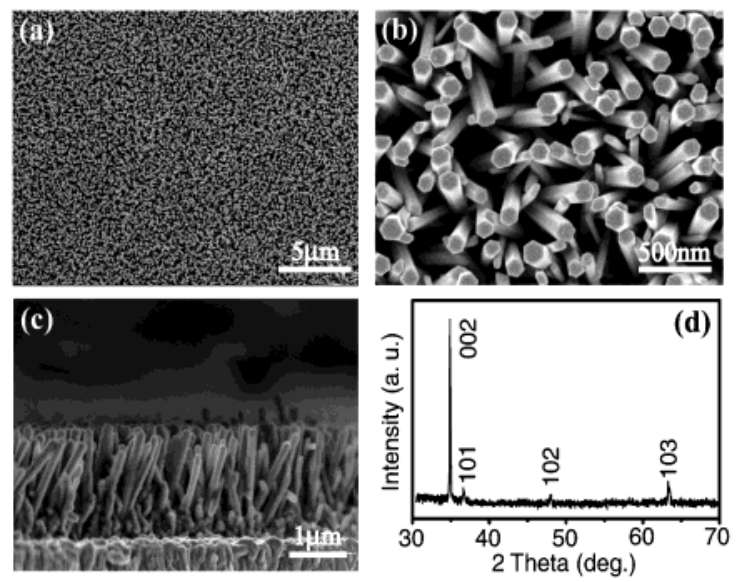

Figure 1. (a, b) FE-SEM top-images of the as-prepared $\mathrm{ZnO}$ nanorod films at low and high magnifications, respectively. (c) Cross-sectional view of the aligned $\mathrm{ZnO}$ nanorods. (d) XRD pattern of the as-synthesized nanorod films.
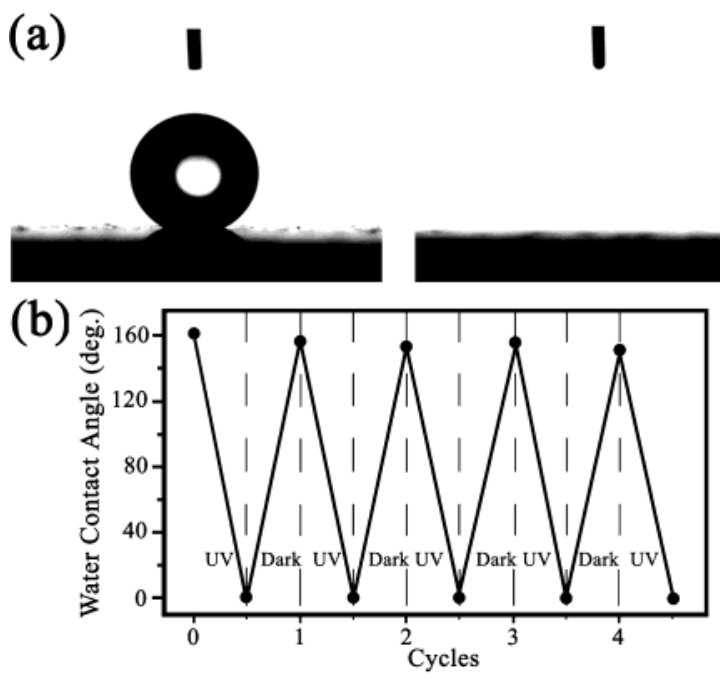

Figure 2. (a) Photographs of water droplet shape on the aligned $\mathrm{ZnO}$ nanorod films before (left) and after (right) UV illumination. (b) Reversible super-hydrophobic-super-hydrophilic transition of the as-prepared films under the alternation of UV irradiation and dark storage.

$\pm 10 \mathrm{~nm}$ ) irradiation for $2 \mathrm{~h}$, the water droplet spread out on the film, resulting in a CA of about $0^{\circ}$ (Figure $2 \mathrm{a}$, right). These results indicate that the wettability changes from super-hydrophobicity to super-hydrophilicity. After the UV irradiated films were placed in the dark for 7 days, a new water droplet was used to measure the surface wettability, and the super-hydrophobicity of the nanorod films was obtained again. This process has been repeated several times, and good reversibility of the surface wettability was observed (shown in Figure 2b). 
To thoroughly understand the reversible super-hydrophobicity to super-hydrophilicity transition of the aligned $\mathrm{ZnO}$ nanorod films, the surface free energy and the surface roughness, which are two main factors governing the surface wettability, are considered. It is well-known that only when the surface free energies of the various crystallographic planes differ significantly could an anisotropic nanorod growth be realized, ${ }^{12}$ and a fast growing plane generally tends to disappear leaving behind slower growing planes with lower surface energy. For the anisotropic $\mathrm{ZnO}$ nanorod, the velocities of crystal growth in different directions were reported to be [-100] $>[-101]>[001] \approx[00-1] \cdot{ }^{13}$ Figure 1 show that the $\mathrm{ZnO}$ nanorods are almost vertically oriented with the well-faceted (001) end projecting out. Compared with other random orientation of $\mathrm{ZnO}$ nanocrystal films, ${ }^{14,15}$ the as-prepared films have the lowest surface free energy. Figure 1 also shows that the nanorods grow separately on the substrate, and air can be present in the troughs between individual nanorods. The hydrophobicity of a rough surface can be intensified by increasing the proportion of air/water interface, ${ }^{1 b}$ e. g., super-hydrophobic surfaces have been successfully fabricated by the aligned carbon nanotubes and polymer nanofibers. ${ }^{4}$ The nanorods and air composite rough surface structure in this case can also greatly enhance the hydrophobicity of the films. Accordingly, both the lower surface free energy and the higher surface roughness contribute to the super-hydrophobicity of the as-prepared films.

As reported, ${ }^{15} \mathrm{UV}$ irradiation will generate electron-hole pairs in the $\mathrm{ZnO}$ surface, and some of the holes can react with lattice oxygen to form surface oxygen vacancies. Meanwhile, water and oxygen may compete to dissociatively adsorb on them. The defective sites are kinetically more favorable for hydroxyl adsorption than oxygen adsorption. As a result, the surface hydrophilicity is improved, and the water $\mathrm{CA}$ of a relative flat $\mathrm{ZnO}$ surface changes from $109^{\circ}$ to $5^{\circ} .{ }^{15}$ For a rough surface, water will enter and fill the grooves of the films, leaving only the up part of the nanorods not in contact with the liquid, which is the three-dimensional capillary effect of a rough surface. ${ }^{6}$ This effect results in a water CA of about $0^{\circ}$. Namely, combining with the rough structure, the UV irradiation causes the surface wettability of aligned $\mathrm{ZnO}$ nanorod films to change from super-hydrophobicity to super-hydrophilicity.

It has been demonstrated that the surface becomes energetically unstable after the hydroxyl adsorption. While the oxygen adsorption is thermodynamically favored, and it is more strongly bonded on the defect sites than the hydroxyl group. ${ }^{15} \mathrm{~A}$ similar result has also been observed on the $\mathrm{TiO}_{2}$ surface. ${ }^{5 b, 16}$ Therefore, the hydroxyl groups adsorbed on the defective sites can be replaced gradually by oxygen atoms when the UV-irradiated films were placed in the dark. Subsequently, the surface evolves back to its original state (before UV irradiation), and the wettability is reconverted from super-hydrophilicity to super-hydrophobicity.

On the basis of the above analysis, it can be concluded that the reversible switching between super-hydrophilicity and superhydrophobicity is related to the cooperation of the surface chemical composition and the surface roughness. The former provides a photosensitive surface, which can be switched between hydrophilicity and hydrophobicity, and the latter further enhances these properties. In addition, the reversible conversions of the surface wettability proceed only by the adsorption and desorption of surface hydroxyl groups at the outmost layer of oxide films, ${ }^{5 b}$ while the structure below the outmost layer remains stable, free from changes in chemical conditions. Therefore, it is reasonable that the reversible wettability switching properties of the as-prepared films exhibit long-term durability.

In summary, the wettability of aligned $\mathrm{ZnO}$ nanorod films was investigated. Reversible super-hydrophobicity to super-hydrophilicity transition was observed and intelligently controlled by alternation of UV illumination and dark storage. This reversible transition of surface wettability is a completely new concept for preparing smart films. This strategy can be extended to other stimuli-responsive surfaces with similar nanostructure and higher stability, which is certainly significant for future industrial applications.

Acknowledgment. The authors thank the State Key Project for Fundamental Research (G1999064504) and the Special Research Foundation of the National Natural Science Foundation of China (29992530, 20/25/02) for continuing financial support.

\section{References}

(1) (a) Wenzel, R. N. Ind. Eng. Chem. 1936, 28, 988-994. (b) Cassie, A. B. D.; Baxter, S. Trans. Faraday Soc. 1944, 40, 546-551.

(2) Parker, A. R.; Lawrence, C. R. Nature 2001, 414, 33-34.

(3) (a) Onda, T.; Shibuichi, S.; Satoh, N.; Tsujii, K. Langmuir 1996, 12, $2125-$ 2127. (b) Öner, D.; McCarthy, T. J. Langmuir 2000, 16, 7777-7782. (c) Erbil, H. Y.; Demirel A. L.; Avc1 Y.; Mert, O. Science 2003, 299, 13771380 .

(4) Feng, L.; Li, S. H.; Li, Y. S.; Li, H. J.; Zhang, L. J.; Zhai, J.; Song, Y. L.; Liu, B. Q.; Jiang, L.; Zhu, D. B. Adv. Mater. 2002, 14, 1857-1860.

(5) (a) Wang, R.; Hashimoto, K.; Fujishima, A.; Chikuni, M.; Kojima, E.; Kitamura, A.; Shimohigoshi, M.; Watanabe, T. Nature 1997, 388, 431432. (b) Wang, R.; Sakai, N.; Fujishima, A.; Watanabe, T.; Hashimoto, K. J. Phys. Chem. B 1999, 103, 2188-2194.

(6) (a) Bico, J.; Tordeux, C.; Quéré, D. Europhys. Lett. 2001, 55, 214-220. (b) Bico, J.; Thiele, U.; Quéré, D. Colloids Surf., A 2002, 206, 41-46.

(7) (a) Ichimura, K.; Oh, S. K.; Nakagawa, M. Science 2000, 288, 16241626. (b) Lahann, J.; Mitragotri, S.; Tran, T.; Kaido, H.; Sundaram, J.; Choi I. S.; Hoffer, S.; Somorjai, G. A.; Langer, R. Science 2003, 299, 371-374. (c) Liang, L.; Feng, X.; D.; Liu, J.; Rieke, P. C.; Fryxell, G. E. Macromolecules 1998, 31, 7845-7850. (d) Anastasiadis, S. H.; Retsos, H.; Pispas, S.; Hadjichristidis, N.; Neophytides, S. Macromolecules 2003. 36, 1994-1999.

(8) (a) Tang, Z. K.; Wong, G. K. L.; Yu, P.; Kawasaki, M.; Ohtomo, A.; Koinuma, H.; Segawa, Y. Appl. Phys. Lett. 1998, 72, 3270-3272. (b) Pan, Z. W.; Dai, Z. R.; Wang, Z. L. Science 2001, 291, 1947-1949. (c) Huang, M. H.; Mao, S.; Feick, H.; Yan, H.; Wu, Y.; Kind, H.; Weber, R.; Russo, R.; Yang, P. Science 2001, 292, 1897-1895.

(9) (a) Tian, Z. R.; Voigt, J. A.; Liu, J.; Mckenzie, B.; Mcdermott, M. J. J. Am. Chem. Soc. 2002, 124, 12954-12955. (b) Greene, L. E.; Law, M.; Goldberger, J.; Kim, F.; Johnson, J. C.; Zhang, Y.; Saykally, R. J.; Yang, P. Angew. Chem., Int. Ed. 2003, 42, 3031-3034.

(10) Sakohara, S.; Tickanen, L. D.; Anderson, M. A. J. Phys. Chem. 1992 96, 11086-11091.

(11) Fujihara, S.; Sasaki, C.; Kimura, T. Appl. Surf. Sci. 2001, 180, 341-350.

(12) Puntes, V. F.; Krishnan, K. M.; Alivisatos, A. P. Science 2001, 291, $2115-$ 2117.

(13) Vayssieres, L.; Keis, K.; Hagfeldt, A.; Lindquist, S. E. Chem. Mater. 2001, 13, 4395-4398.

(14) Li, M.; Zhai, J.; Liu, H.; Song, Y. L.; Jiang, L.; Zhu, D. B. J. Phys. Chem. B 2003, 107, 9954-957.

(15) Sun, R. D.; Nakajima, A.; Fujishima, A.; Watanabe, T.; Hashimoto, K. J. Phys. Chem. B 2001, 105, 1984-1990.

(16) Wang, L. Q.; Baer, D. R.; Engelhard, M. H.; Shultz, A. N. Surf. Sci. 1995, 344, 237-350.

JA038636O 